EPJ Web of Conferences 43, 04008 (2013)

DOI: $10.1051 /$ epjconf/20134304008

(C) Owned by the authors, published by EDP Sciences, 2013

\title{
Improved determination of the atmospheric parameters of the pulsating sdB star Feige 48
}

\author{
M. Latour ${ }^{1, a}$, G. Fontaine ${ }^{1}$, P. Brassard ${ }^{1}$, P. Chayer ${ }^{2}$, and E.M. Green ${ }^{3}$ \\ ${ }^{1}$ Département de Physique, Université de Montréal, Succ. Centre-Ville, CP. 6128, Montréal, \\ QC H3C 3J7, Canada \\ ${ }^{2}$ Space Telescope Science Institute, 3700 San Martin Drive, Baltimore, MD 21218, USA \\ ${ }^{3}$ Steward Observatory, University of Arizona, 933 North Cherry Avenue, Tucson, AZ 85721 , \\ USA
}

\begin{abstract}
Given the importance of Feige 48 as an sdB pulsator, we sought to obtain the best possible estimates of its spectroscopic parameters with a grid of NLTE metal-blanketed model atmospheres constructed especially for that star. This small grid of 150 models includes 8 metallic elements whose abundances have been determined previously and reported in the literature. Our fitting procedure found the following parameters for Feige 48: $T_{\text {eff }}=29504 \mathrm{~K}, \log g=5.41$ and $\log N(\mathrm{He}) / N(\mathrm{H})=-2.90$. These results are in very good agreement with previous spectroscopic estimates (which generally ignore either NLTE effects or metal blanketing), thus indicating that metal line-blanketing in NLTE - modeled for the first time here - is not a dominant factor in the atmosphere of Feige 48.
\end{abstract}

\section{ASTROPHYSICAL CONTEXT}

Feige 48 is a well known rapidly pulsating subdwarf $\mathrm{B}(\mathrm{sdB})$ star. Asteroseismic analyses carried out on this star have found it, among other things, to have a mass of $0.46 M_{\odot}$ [1]. The synchronous rotation between Feige 48 and its invisible companion (most likely a white dwarf) forming a binary system with an orbital period of $9.02 \mathrm{~h}$ has also been established using asteroseismology [2]. Both of these important results rely on independent estimates of the effective temperature and surface gravity provided by spectroscopic analyses.

These analyses are usually made by fitting simultaneously the Balmer and helium lines of an observed spectrum with a grid of model atmospheres. It is then possible to find the best combination of parameters that reproduce the spectrum of the star. As for Feige 48, its parameters have been determined with different types of models atmospheres (LTE, LTE with metals, NLTE) by differents studies and they all gave similar results: a $T_{\text {eff }}$ between 29 and $30 \mathrm{kK}, \log g \sim 5.4-5.5$ and $\left.\log N(\mathrm{He}) / N(\mathrm{H})\right) \sim-2.9$ [3] [4] [1]. In this work, we want to redetermine these parameters by using state-of-the-art NLTE model atmospheres that also include the line-blanketing effect of metallic elements.

\section{THE CHEMICAL COMPOSITION AND ATMOSPHERIC PARAMETERS OF FEIGE 48}

In order to fix a suitable chemical composition for our grid of model atmospheres, we relied on two abundance studies that have been done for Feige 48. The abundances of the first study we used were determined by using Keck HIRES optical spectra [4]. As for the second study, it used UV HST/STIS spectroscopy [5]. Their results are summarized in Table 1 where we also give the average abundance for each element. The abundances for the elements we included in our models are in bold.

\footnotetext{
ae-mail: marilyn@astro.umontreal.ca
}

This is an Open Access article distributed under the terms of the Creative Commons Attribution License 2.0, which permits unrestricted use, distribution, and reproduction in any medium, provided the original work is properly cited. 
Table 1. Chemical composition of Feige 48.

\begin{tabular}{lccc}
\hline & \multicolumn{3}{c}{ Abundances $(\log N(\mathrm{X}) / N(\mathrm{H}))$} \\
Elements & {$[4]$} & {$[5]$} & Mean \\
\hline Carbon & -4.64 & -4.91 & $\mathbf{- 4 . 7 5}$ \\
Nitrogen & -4.33 & -4.39 & $\mathbf{- 4 . 3 6}$ \\
Oxygen & -4.20 & - & $-\mathbf{4 . 2 0}$ \\
Neon & -4.90 & - & -4.90 \\
Magnesium & -5.10 & - & -5.10 \\
Aluminium & -6.50 & -6.49 & -6.50 \\
Silicon & -5.70 & -5.47 & $\mathbf{- 5 . 5 7}$ \\
Phosphorus & - & -7.59 & $\mathbf{- 7 . 5 9}$ \\
Sulfur & -5.85 & - & $\mathbf{- 5 . 8 5}$ \\
Iron & -4.45 & -4.29 & $\mathbf{- 4 . 3 6}$ \\
Nickel & - & -5.31 & $\mathbf{- 5 . 3 1}$ \\
\hline
\end{tabular}

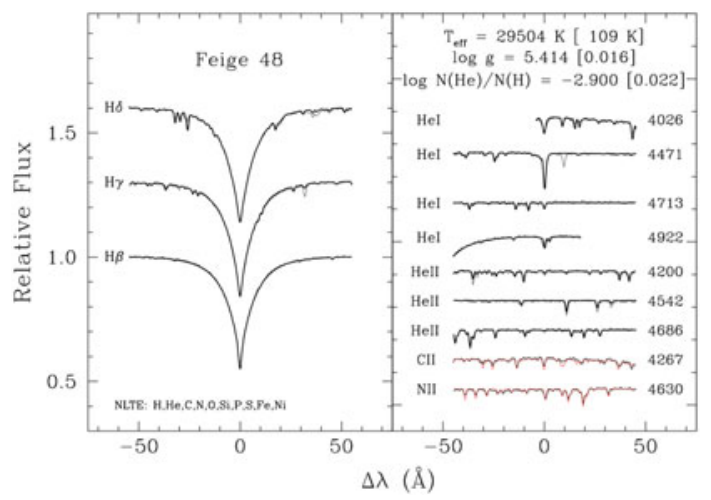

Figure 1. Resulting fit and parameters for Feige 48.

Our grid of NLTE metal line-blanketed model atmospheres are computed with the public codes TLUSTY and SYNSPEC. The grid for Feige 48 include 5 values of $T_{\text {eff }}, 6$ values of $\log g$, and 5 different helium abundances, all of which obviously centered on the parameters of the star. Figure 1 shows our resulting fit and parameters for Feige 48. Our observed spectrum has a high $\mathrm{S} / \mathrm{N}(\sim 400)$ and a resolution of $\sim 1 \AA$. We can see that the Balmer and He I lines are very well reproduced and the metallic features seen are mostly O II, N II, Fe III and C II lines. Since the abundances we used were made with different kinds of model atmospheres than ours, we decided to compare our final model with the metallic features seen in the UV spectrum of Feige 48. Overall, the metal lines in the optical and the UV are well reproduced by our synthetic spectrum, though the match could be improved by fine-tuning the metal abundances.

\section{CONCLUSION}

Our spectroscopic analysis of Feige 48 leads to parameters similar to those already found in the literature on the basis of less sophisticated models. This means that NLTE metal line-blanketing does not significantly affect the optical spectrum of the star. It also implies that these effects should be very small for sdB stars with effective temperature around $30000 \mathrm{~K}$. With at least three different studies now, made with different types of model atmospheres, and leading to similar atmospheric parameters for Feige 48, these values must certainly be considered as fully reliable.

The comparison of metal lines in the spectra of Feige 48 shows a relatively good agreement with our final model atmosphere of the star. Some fine-tuning of the abundances could still improve the match between observations and predictions, but it would not change significantly the inferred chemical composition nor the atmospheric parameters of the star. At the effective temperature and surface gravity of Feige 48, NLTE effects do not seem to affect the metallic UV lines in an important way. This is a good thing since abundance analyses of sdB stars are usually done with LTE models and, therefore, their results should remain reliable within a certain (not too large) uncertainty range.

\section{References}

[1] Charpinet, S., Fontaine, G., Brassard, P., et al. 2005, A\&A, 443, 251

[2] Van Grootel, V., Charpinet, S., Fontaine, G., \& Brassard, P. 2008, A\&A, 483, 875

[3] Koen, C., O’Donoghue, D., Pollacco, D.L., \& Nitta, A. 1998, MNRAS, 300, 1105

[4] Heber, U., Reid, I.N., \& Werner, K. 2000 A\&A, 363,198

[5] O'Toole, S.J., \& Heber, U. 2006, A\&A, 452, 579 